\title{
Corrigendum
}

\section{Corrigendum to "Reduction of Residual Stress for High-Strength Low-Alloy Steel Strip Based on Finite Element Analysis"}

\author{
Zengshuai Qiu (D), ${ }^{1}$ Anrui He, ${ }^{1}$ Jian Shao ${ }^{D},{ }^{1}$ and Xiaoming Xia ${ }^{2}$ \\ ${ }^{1}$ National Engineering Research Center for Advanced Rolling, University of Science and Technology Beijing, \\ Beijing 100083, China \\ ${ }^{2}$ Baosteel Shanghai Meishan Iron \& Steel Co. Ltd., Nanjing 210039, China \\ Correspondence should be addressed to Jian Shao; ustbshao@163.com
}

Received 1 March 2018; Accepted 5 March 2018; Published 1 April 2018

Copyright ( 12018 Zengshuai Qiu et al. This is an open access article distributed under the Creative Commons Attribution License, which permits unrestricted use, distribution, and reproduction in any medium, provided the original work is properly cited.

In the article titled "Reduction of Residual Stress for HighStrength Low-Alloy Steel Strip Based on Finite Element Analysis" [1], Dr. Zengshuai Qiu was incorrectly listed as the corresponding author. The corresponding author is Dr. Jian Shao.

\section{References}

[1] Z. S. Qiu, A. R. He, J. Shao, and X. M. Xia, "Reduction of residual stress for high-strength low-alloy steel strip based on finite element analysis," Advances in Materials Science and Engineering, vol. 2018, Article ID 8131909, 13 pages, 2018. 


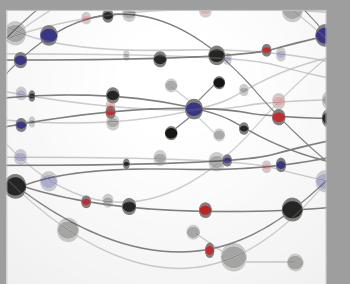

The Scientific World Journal
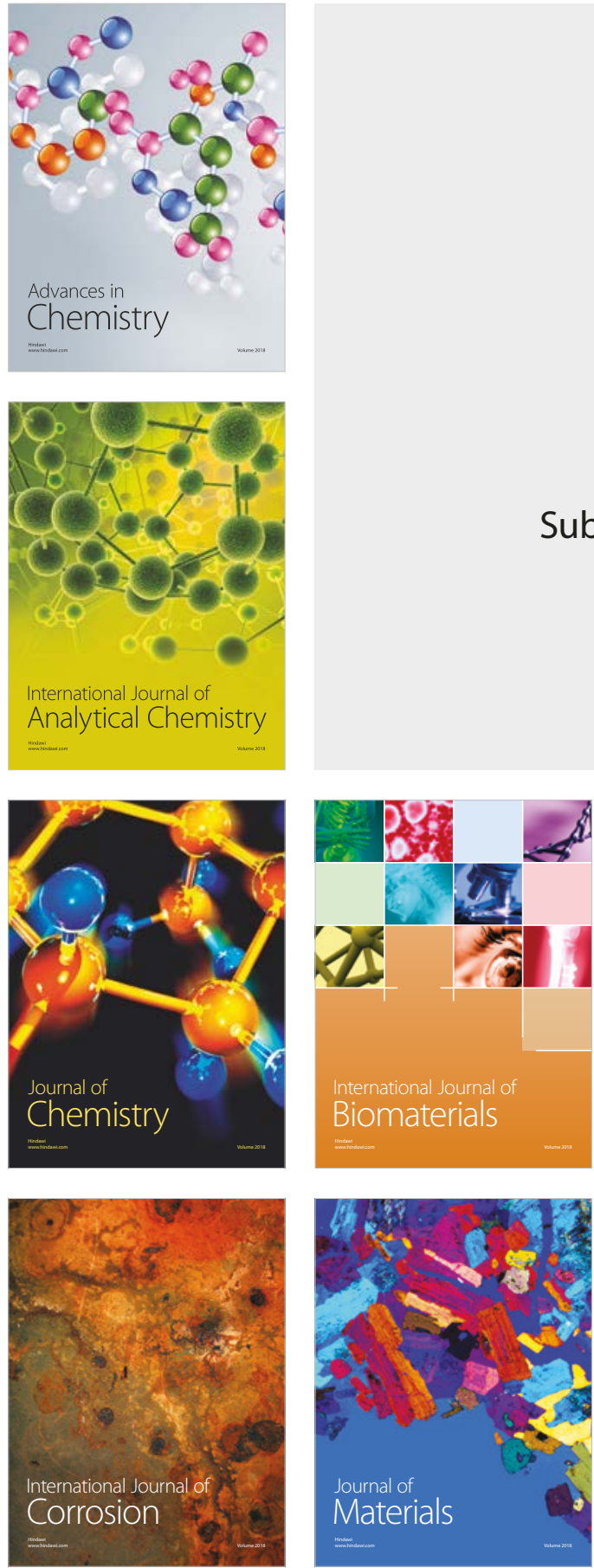

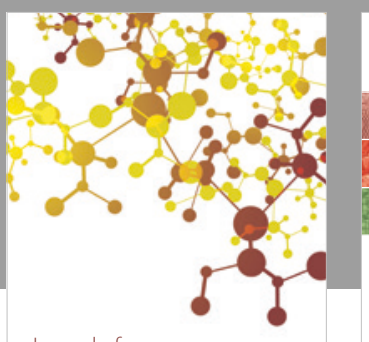

Journal of

Applied Chemistry
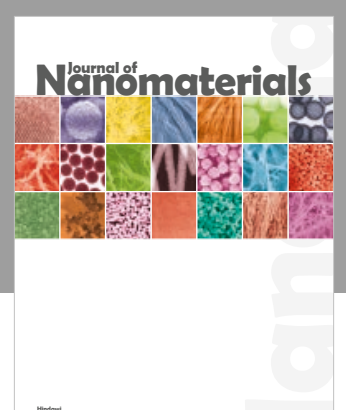

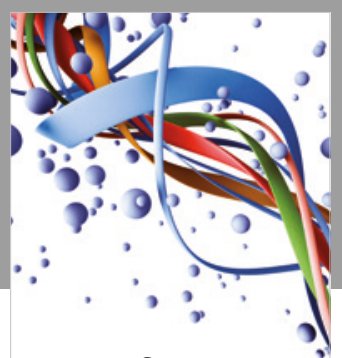

Scientifica

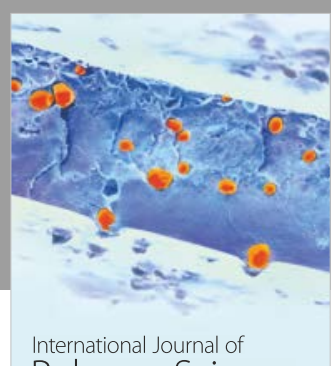

Polymer Science

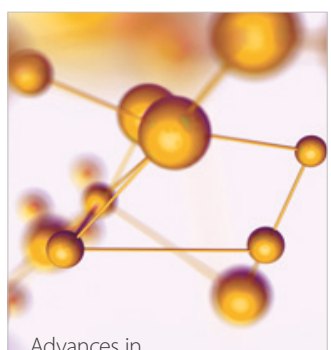

Physical Chemistry
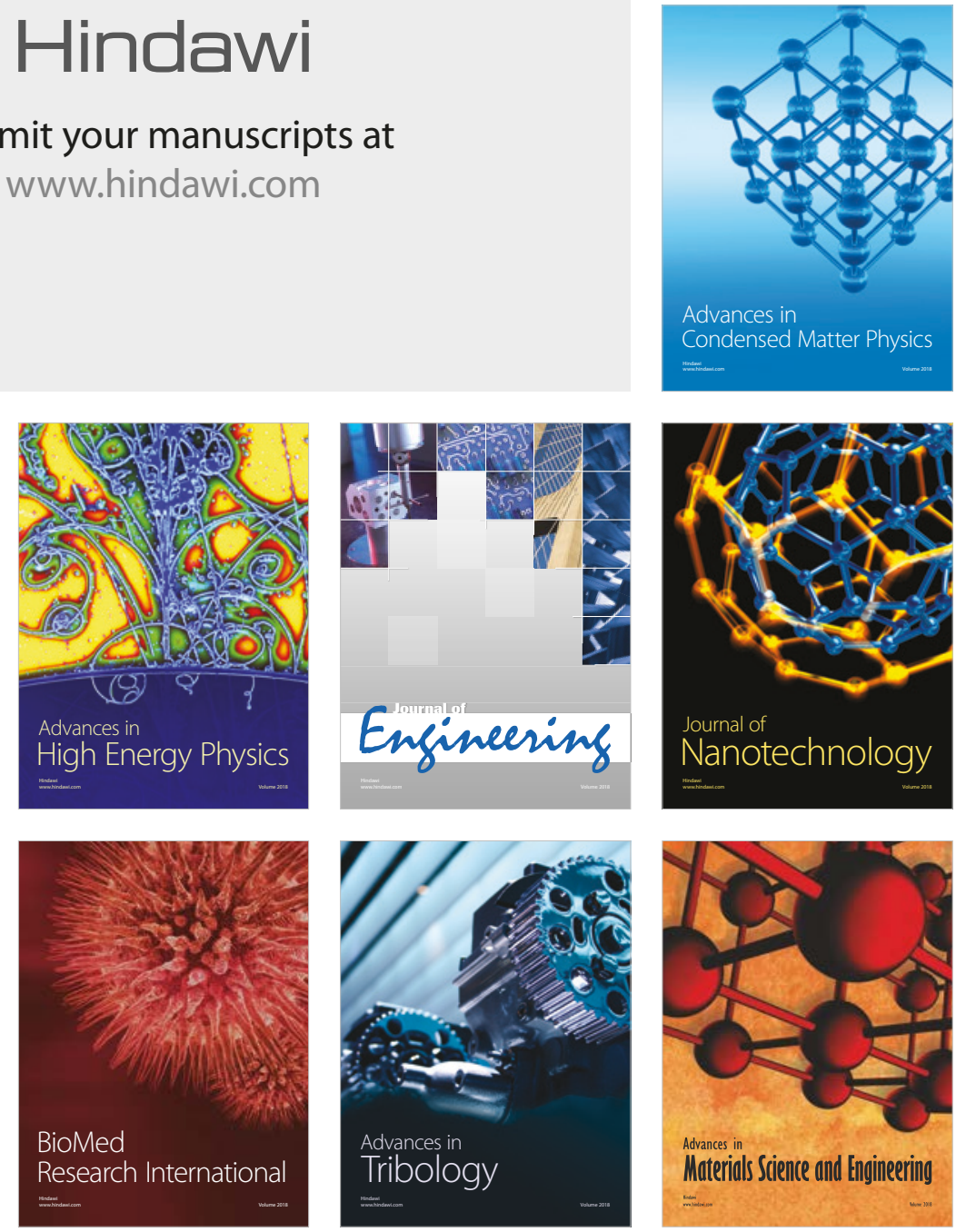